\title{
GRADIENTES DE SALINIDADE E FREQÜÊNCIA DE ALAGAMENTO COMO DETERMINANTES DA DISTRIBUIÇÃO E BIOMASSA DE MACROALGAS ASSOCIADAS A TRONCOS DE MANGUEZAIS NA BAÍA DE BABITONGA, SC
}

\author{
CUNHA, S. R. ${ }^{1} \&$ C.S.B. COSTA ${ }^{2}$ \\ ${ }^{1}$ Laboratório de Ecologia da Vegetação Costeira - CTTMar/UNIVALI, Itajaí, SC. e-mail: \\ simone@cttmar.univali.br \\ ${ }^{2}$ Depto. Oceanografia, Fundação Universidade Federal do Rio Grande (FURG), Rio \\ Grande, RS
}

\begin{abstract}
RESUMO
Este trabalho teve como objetivo avaliar a influência dos gradientes de salinidade e freqüência de alagamento sobre a distribuição e biomassa das comunidades de macroalgas associ-

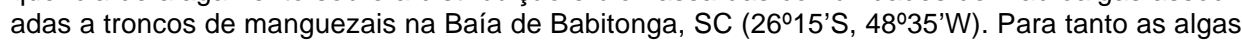
foram coletadas ao longo dos troncos em faixas de $10 \mathrm{~cm}$ desde o substrato até o limite superior de distribuição das algas, em manguezais próximos à margem, desde a porção mais oligohalina até a porção euhalina da Baia da Babitonga. Este material foi separado por espécie, seco em estufa até a obtenção de peso constante, e representado como biomassa de algas por área de tronco $(\mathrm{g}$ $\mathrm{ms} \mathrm{m}^{-2}$ ). Para avaliar a variação das comunidades, foram utilizadas análises de agrupamento e PCA, considerando a biomassa por espécie em cada freqüência de alagamento e em cada estação amostral. A biomassa média de macroalgas na porção euhalina $(195,9 \pm 58,8 \mathrm{~g}$ ms (massa seca) $\mathrm{m}^{-2}$ por área de tronco) foi significativamente superior à da porção oligohalina da Baía $(36,9$ $\pm 13,5 \mathrm{~g} \mathrm{~ms} \mathrm{~m}^{-2}$ ) e, embora não tenha sido observada diferença significativa da biomassa das estações mesohalinas com as estações oligo e euhalinas, houve uma correlação positiva entre biomassa de macroalgas e salinidade $\left(r^{2}=0,83\right)$. Através de análise de agrupamento e PCA foi possível identificar a formação dos seguintes grupos: 1) região euhalina com frequêencia de alagamento superior a $92 \%$ do tempo, dominada por Bostrychia montagnei; 2) região euhalina com freqüência de alagamento inferior a $92 \%$ do tempo, dominada por Bostrychia tenella; 3) região mesohalina, dominada por Bostrychia calliptera e Bostrychia pinnata; 4) região oligohalina dominada por Catenella caespitosa. Tanto o grupo constituído pelas estações mesohalinas como o constituído pelas oligohalinas foram divididos em subgrupos determinados por freqüências de alagamento maiores ou menores que $92 \%$. Além das diferenças em termos de biomassa e espécie dominante, houve uma redução significativa na diversidade da região euhalina (15 espécies) para a região oligohalina (4 espécies). A consistência na formação de grupos de estações e níveis intermareais amostrados, evidenciou a importância da salinidade e do alagamento como estruturadores das comunidades de macroalgas de manguezais na Baía da Babitonga.
\end{abstract}

Palavras-chave: macroalgas de manguezais, biomassa, zonação, Bostrychia

\section{SALINITY AND FLOODING FREQUENCY GRADIENTS AS DETERMINANT OF SEAWEED DISTRIBUTION AND BIOMASS ON TRUNKS AND ROOTS OF MANGROVE TREES IN BABITONGA BAY,SC}

\footnotetext{
ABSTRACT

This work aimed to evaluate the influence of salinity and flooding frequency on distribution and biomass of seaweed communities occurring on trunks and roots of mangrove trees in Babitonga

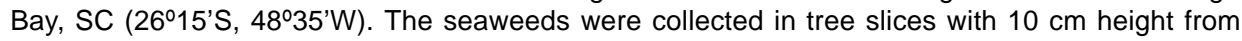




\begin{abstract}
the bottom to its upper limit on the trees, in oligohaline, mesohaline e euhalinde areas of the bay. The seaweeds were sorted by species, dried until constant weight, and presented as algae biomass per trunk area ( $\mathrm{g} \mathrm{dm}$ (dry mass) $\mathrm{m}^{-2}$ of substrate). Cluster analysis was applied to study these communities, using biomass of each species in each flooding level, in each site of the bay. The average biomass in euhaline place $\left(195,9 \pm 58,8 \mathrm{~g} \mathrm{dm} \mathrm{m}^{-2}\right)$ was significantly higher than in oligohaline place $\left(36,9 \pm 13,5 \mathrm{~g} \mathrm{dm} \mathrm{m}^{-2}\right)$. There was no significant difference in biomass from mesohaline to euhaline, neither from mesohaline to oligohaline, but a positive correlation occurred between biomass and salinity $\left(r^{2}=0,83\right)$. In the cluster analysis the groups formed were: 1$)$ euhaline site with flooding frequency higher than $92 \%$ of time, dominated by Bostrychia montagnei; 2) euhaline site with flooding frequency lower than $92 \%$ of time, dominated by Bostrychia tenella; 3 ) mesohaline site, dominated by Bostrychia calliptera and Bostrychia pinnata; 4) oligohaline site, dominated by Catenella caespitosa. The mesohaline group, as much as the oligohaline group was spitted in smaller groups with flooding frequencies lower or higher than $92 \%$ of time. Differences were also observed in diversity, and number of species was higher in euhaline site (15 species) and lower in olighaline site (4 species). The results of this works show how important salinity and flooding frequency are to structure of mangrove seaweeds in Babitonga Bay.
\end{abstract}

Key-words: mangrove seaweeds, biomass, zonation, Bostrychia

\section{INTRODUÇÃO}

A associação de macroalgas epifíticas denominada por Post (1968) de Bostrychietum encontra nos troncos das árvores de mangue um substrato adequado à sua ocupação. Tendo em vista que colonizam o ambiente estuarino intermareal, estas macroalgas estão sujeitas a variações de salinidade devido à descarga fluvial, variações na irradiância, na temperatura, no nível de nutrientes e de dessecação, devido aos ciclos de submersão e emersão conseqüentes das oscilações da maré (Mann \& Steinke, 1988; Dawes 1996). Estas flutuações ambientais podem influenciar nos padrões de distribuição horizontal e vertical das macroalgas, modificando a estrutura das comunidades e a dinâmica de produção de biomassa ao longo do ambiente estuarino.

A tolerância destas algas às variações de salinidade permite que elas ocupem uma grande variedade de habitats, mas geralmente há um aumento, tanto na biomassa quanto na diversidade destas macroalgas, com o aumento de salinidade. As espécies do gênero Bostrychia, especialmente $B$. radicans, além de serem bastante tolerantes às variações de salinidade (Mann \& Steinke, 1988; Karsten et al., 1994a,b; Broderick \& Dawes, 1998), são capazes de ocupar áreas sujeitas a elevados períodos de emersão, e conseqüentemente, elevada dessecação. Esta tolerância à dessecação vem sendo demonstrada em vários estudos sobre a distribuição vertical, tanto no Brasil (Eston et al., 1992; Cultrim, 1998; Yokoya et al., 1999; Bouzon \& Ouriques, 1999), como em outros manguezais ao longo do mundo (Peña, 1998; Mann \& Steinke, 1988; Aikanathan \& Sasekumar, 1994; Phillips et al. 1996). Vários estudos fisiológicos (Mann \& Steinke, 1988; Broderick \& Dawes, 1998; Peña et al., 1999) confirmam a interpretação ecológica de ampla tolerância do gênero Bostrychia sugerida pelos estudos de distribuição vertical.

Existe hoje grande interesse sobre a distribuição das macroalgas associadas a manguezais assim como sobre sua ampla tolerância fisiológica a variações de salinidade e dessecação ( Karsten et al., 1994a,b; Broderick \& Dawes 1998; Peña et al., 1999), especialmente porque esta tolerância influencia diretamente sobre a produtividade das algas. A ampla tolerância das algas de manguezal à condições tipicamente estressantes do ambiente estuarino e intermareal, além da capacidade de produção líquida mesmo em períodos de emersão (Mann \& Steinke, 1988; Peña et al., 1999) sugerem que estes organismos podem representar uma importante fonte de carbono para os manguezais. 
Este trabalho teve como objetivo avaliar a influência dos gradientes de salinidade e freqüência de alagamento sobre a distribuição e biomassa das comunidades de macroalgas associadas a troncos de manguezais na Baía de Babitonga, SC.

\section{METODOLOGIA}

A distribuição espacial das macroalgas associadas a troncos de mangue foi avaliada em quatro estações amostrais ao longo da Baía da Babitonga, SC (2612'S e 48 $46^{\circ} \mathrm{W}$, Figura 1, áreas 1, 3, 4 e 8). Em cada estação foi definida uma área de amostragem com $1000 \mathrm{~m}^{2}$ (100 m paralelamente à margem e 10 m perpendicularmente), próximo à margem do manguezal e com quota estimada em 0,7 m acima do nível zero de maré baixa. Dados de salinidade foram obtidos em cada estação em diferentes épocas do ano, e as médias anuais em estudos prévios (IBAMA, 1998).

Durante o período de verão (janeiro de 1998), em cada área de manguezal as algas presentes em cinco troncos das árvores escoIhidas aleatoriamente (tabela de números aleatórios) foram coletadas através da raspagem da superfície de ocorrência, utilizando-se amostradores de $25 \mathrm{~cm}^{2}$ (2,5 cm de largura por $10 \mathrm{~cm}$ de altura), ao longo do comprimento do tronco, segundo a estratificação devido ao nível de alagamento (Figura 2).

A freqüência de alagamento em cada nível topográfico foi calculada utilizando-se a posição (altura) do nível com relação ao nível zero de maré baixa estabelecido pela tábua de maré da Capitania dos Portos para o Porto de São Francisco do Sul, e observações de campo. A seguir foram calculados o número de dias de emersão contínua a que cada nível está sujeito entre o período de duas marés de sizígia consecutivas, ou seja, quantos dias a planta fica continuamente submersa a cada 15 dias (Figura 2).

As macroalgas coletadas foram acondicionadas em sacos plásticos e conduzidas ao laboratório, onde as amostras foram congeladas. Durante o processamento o material foi lavado, separado por espécie em microscópio estereoscópico, seco em estufa a $60^{\circ} \mathrm{C}$ até atingir peso constante e pesado em balança analítica. A biomassa de macroalgas associadas aos troncos foi expressa em $\mathrm{g} \mathrm{ms} \mathrm{m}^{-2}$ de tronco (gramas de massa seca de algas por $\mathrm{m}^{2}$ de tronco). O perímetro das árvores $(\mathrm{Cu}-$ nha, 2001) foi multiplicado pela altura do tronco ocupada pela alga para obtenção da área de tronco disponível por $\mathrm{m}^{2}$ de manguezal $\left(\mathrm{m}^{2} /\right.$ $\mathrm{m}^{2}$ ). Utilizando esta informação, a biomassa por área de tronco foi convertida em biomassa por área de manguezal $\left(\mathrm{g} \mathrm{ms} \mathrm{m}^{-2}\right.$ de manguezal).

\section{RESULTADOS E DISCUSSÃO}

Os dados de salinidade de verão, outono e inverno evidenciam o gradiente de salinidade ao longo do eixo da baía (Tabela 1). Houve um aumento gradual da biomassa média de macroalgas associadas aos troncos nas margens dos manguezais, da estação 1 (região oligohalina) para as estações 3 e 4 (mesohalinas), e um aumento maior da estação 4 para a 8 (euhalina). A biomassa média da estação 8 apresentou-se significativamente diferente da estação $1(p=0,01)$ (Figuras $3 A$ e 3B).

A composição das espécies variou acentuadamente entre as estações localizadas nos extremos de salinidade na Baía da Babitonga (Tabela 2) e estas variações foram refletidas na análise de agrupamento (Figura 4A). Houve a redução do número de espécies do gênero Bostrychia de 8 para 4, da estação 8 (euhalina) para a estação 1 (oligohalina), e Caloglossa leprieurii e C. ogazawaraensis estiveram presentes nas estações das áreas euhalina e mesohalina e ausentes na estação 1 (oligohalina). A redução da diversidade com a redução da salinidade tem sido atribuída à menor tolerância de macroalgas estuarinas a exposições prolongadas a baixas salinidades, 


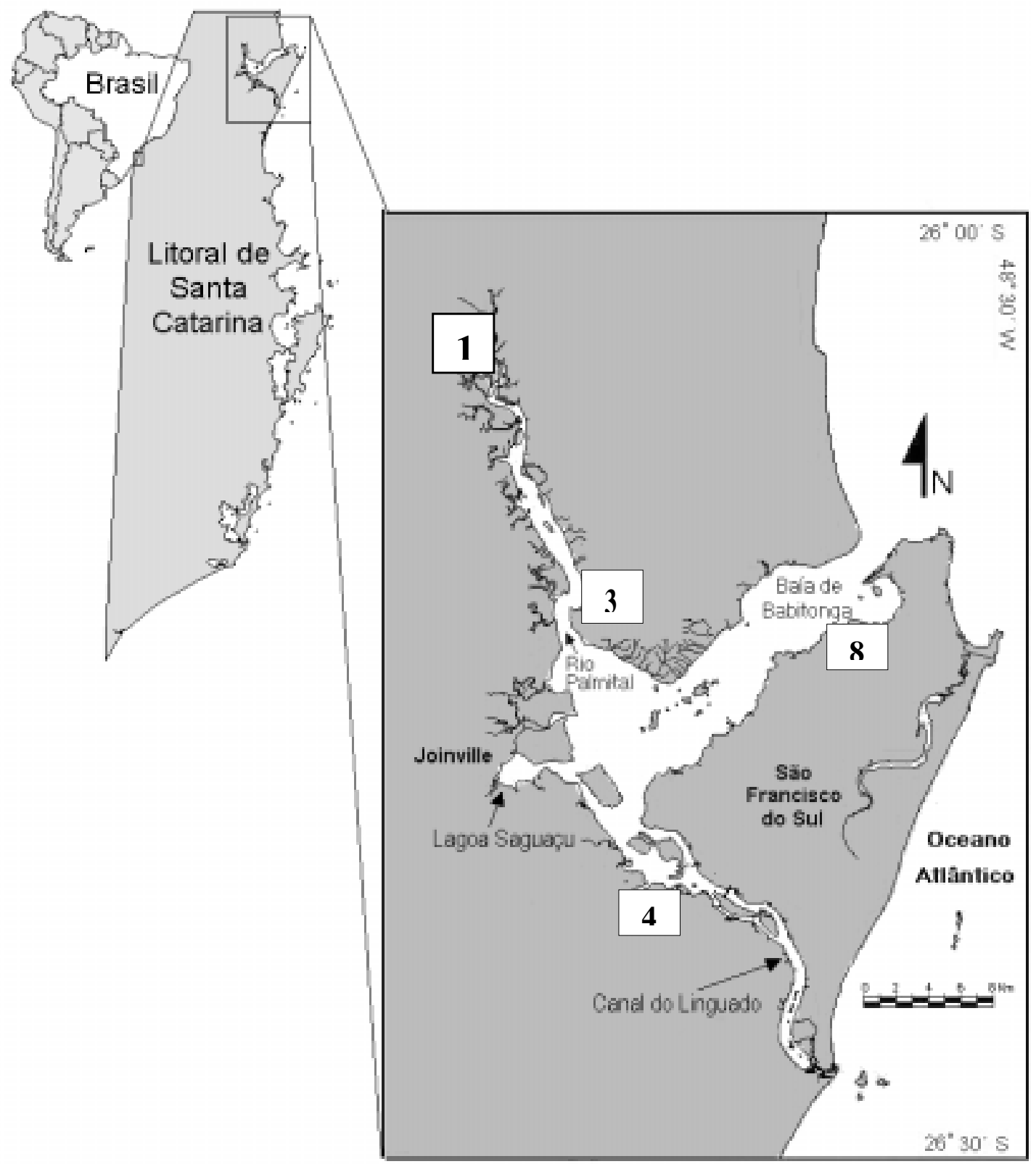

Figura 1: Localização da área de estudo na Baía da Babitonga, Litoral de Santa Catarina, com destaque para as estações 1,3, 4 e 8 , onde as algas foram coletas. 


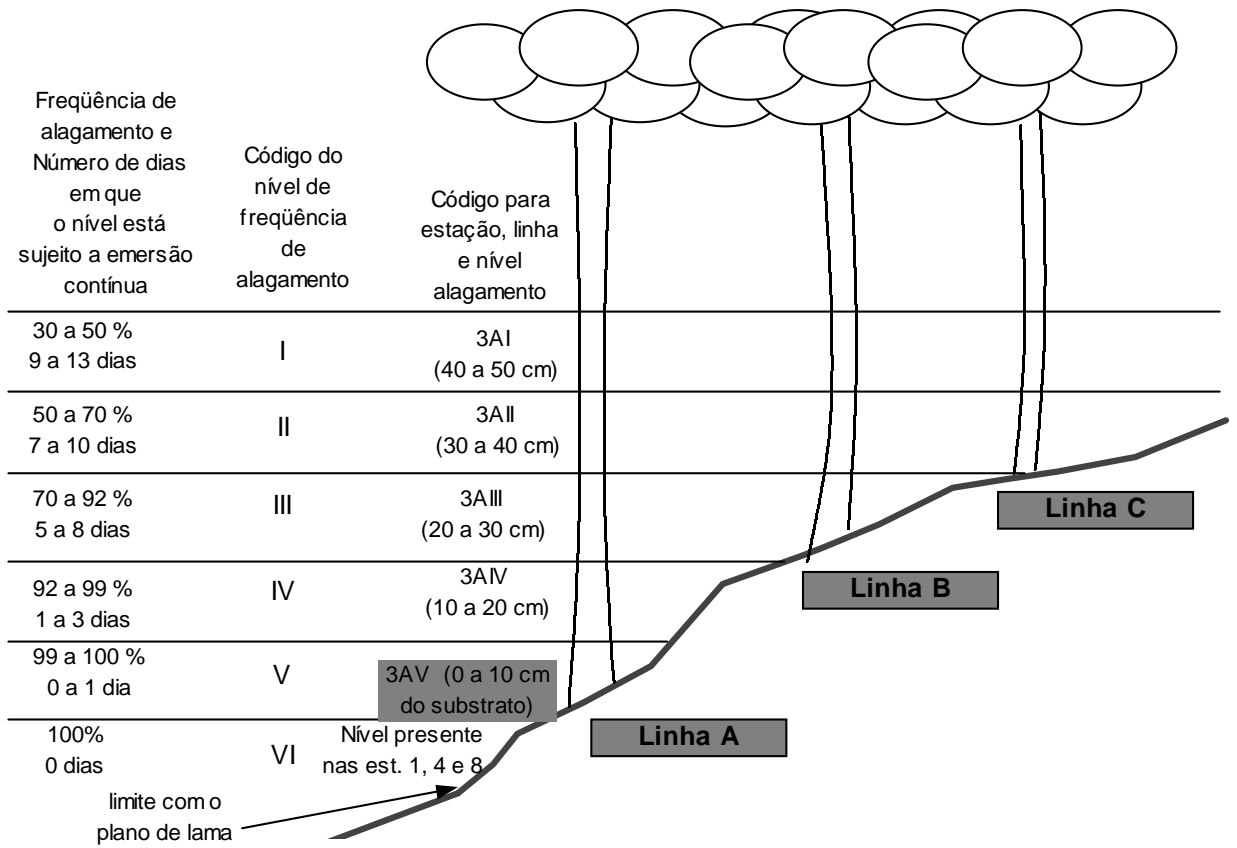

Figura 2: Perfil da estação 3 mostrando a localização das alturas de coleta das macroalgas associadas aos troncos das árvores em cada uma das linhas $(A, B$ e $C)$, com relação à freqüência de alagamento e ao número médio de dias a cada 15 dias, em que cada nível fica continuamente emerso. A freqüência de alagamento representa o número percentual de dias em que um determinado nível sofreu inundação durante a maré alta. Na estação 3 não houve árvores ocupando o nível VI de alagamento. Em frente ao manguezal, após um declive acentuado ocorreu um plano de lama. Nas linhas A das estações 1, 4 e 8, o declive até o plano de lama foi mais suave, permitindo a ocorrência de árvores, e conseqüentemente, macroalgas, no nível VI de alagamento.

mesmo em organismos que apresentem características eurihalinas em experimentos de curta duração (Mann \& Steinke, 1988). Variações da biomassa também foram intensas (Figura 4B). A estação 1 (oligohalina, salinidade 5) apresentou 6 taxa de macroalgas (4 espécies de Bostrychia, Catenella caespitosa e Rhizoclonium) e biomassa média de $2,02 \mathrm{~g} \mathrm{~ms}$ $\mathrm{m}^{-2}$ de manguezal. Já a estação 8 (euhalina, salinidade 30), apresentou 15 taxa (8 espécies de Bostrychia, Caloglossa leprieurii, Polysiphonia howeii, Gelidium, Cladophoropsis membranacea, Bloodleopsis pusilla, Rhizoclonium e Ulvaria) e biomassa média duas vezes maior do que a estação 1 (5,5 $\mathrm{g} \mathrm{ms} \mathrm{m}^{-2}$ de manguezal).

Padrões de zonação são vistos como respostas aos níveis de estresse ao longo da zona intermareal e às interações bióticas, como competição e herbivoria, a que os organismos estão sujeitos (Raffaelli \& Hawkins, 1996; Bertness, 1999). Onde o nível de estresse é maior, o espaço é ocupado por organismos tolerantes ao estresse; onde o ambiente é menos estressante, a distribuição e abundância das espécies sofre influência das interações bióticas. Com o aumento unidirecional do estresse entre os níveis inferior e superior da zona intermareal, há geralmente uma diminuição da biomassa, da produtividade, do recobrimento da área e da riqueza de espécies de origem aquática (Raffaelli \& Hawkins, 1996; Dawes, 1998; Bertness, 1999).

Dentro deste conceito, poderia ser assumido que, na Baía da Babitonga dois importantes gradientes unidirecionais estão atuan- 
CUNHA, S. R. \& C.S.B. COSTA: Macroalgas de manguezais: influência de salinidade e alagamento.

Tabela 1: Variação espacial da salinidade média da água superficial na Baía da Babitonga.

\begin{tabular}{ccccc}
\hline \hline & & Salinidade & \\
\hline Estações & Outono/1997 & & Média Anual $^{\mathrm{a}}$ \\
\hline 1 & 3 & ${\text { Inverno/ } / 1997^{\mathrm{b}}}^{\mathrm{a}}$ & Verão/1998 $^{\mathrm{c}}$ & - \\
3 & 23 & 5 & 0 & $15,5 \pm 5,9$ \\
4 & 28 & 25 & 9 & $25,2 \pm 1,9$ \\
6 & 28 & - & 20 & $22,7 \pm 2,8$ \\
8 & 33 & $29-30^{\mathrm{e}}$ & - & $30,3 \pm 1,6$ \\
\hline \hline
\end{tabular}
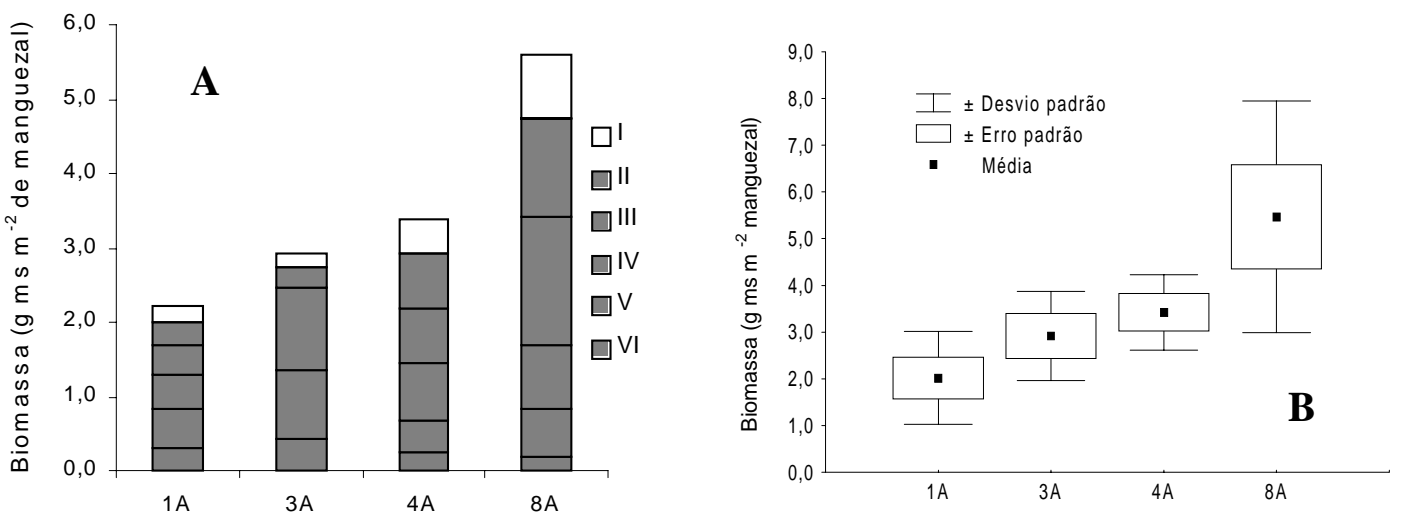

Figura 3: Variação espacial da biomassa média de macroalgas associadas a troncos ( $\mathrm{g} \mathrm{ms} \mathrm{m}^{-2}$ de manguezal) ao longo da Baía da Babitonga na linha A das estações 1 a 8, durante o verão de 1998: (A) biomassa média em cada nível de freqüência de alagamento em cada estação; (B) biomassa média, desvio padrão e erro padrão em cada estação $(n=5)$. Níveis de freqüência de alagamento (percentual de dias em que a maré alta atinge dada altura do tronco): I=30 a 50\%; II=50 a 70\%; III=70 a $92 \%$; IV=92 a $99 \%$; V=99 a $100 \%$; VI=100\%.

Tabela 2: Salinidade da área e freqüência de alagamento na qual a espécie apresentou maiores contribuições para a biomassa do tronco.

\begin{tabular}{lll}
\hline \hline Espécie & Salinidade da área & Freqüência de alagamento \\
\hline Gelidium sp & Euhalina & Altas \\
Ulvaria & Euhalina a mesohalina & Altas \\
Enteromorpha spp & Euhalina a mesohalina & Altas \\
Caloglossa ogazawaraensis & Mesohalina & Altas \\
Caloglossa leprieurii & Mesohalina & Altas \\
B. binderi & Euhalina & Altas e intermediárias \\
B. montagnei & Euhalina a mesohalina & Altas e intermediárias \\
Cladophoropsis membranacea e Bloodleopsis pusilla $*$ & Mesohalina a euhalina & Altas e intermediárias \\
B. radicans f. moniliforme & Oligohalina & Altas e intermediárias \\
Polysiphonia howei & Euhalina a mesohalina & Intermediárias \\
Catenella caespitosa & Oligohalina a mesohalina & Intermediárias \\
B. calliptera e B. pinnata & Mesohalina & Intermediárias a baixas \\
B. tenella & Euhalina a mesohalina & Baixas e intermediárias \\
B. radicans f. radicans & Eurihalina & Baixas e intermediárias \\
Rhizoclonium & Eurihalina & Baixas e intermediárias \\
Cianophyta & Eurihalina & Baixas e intermediárias \\
\hline \hline
\end{tabular}



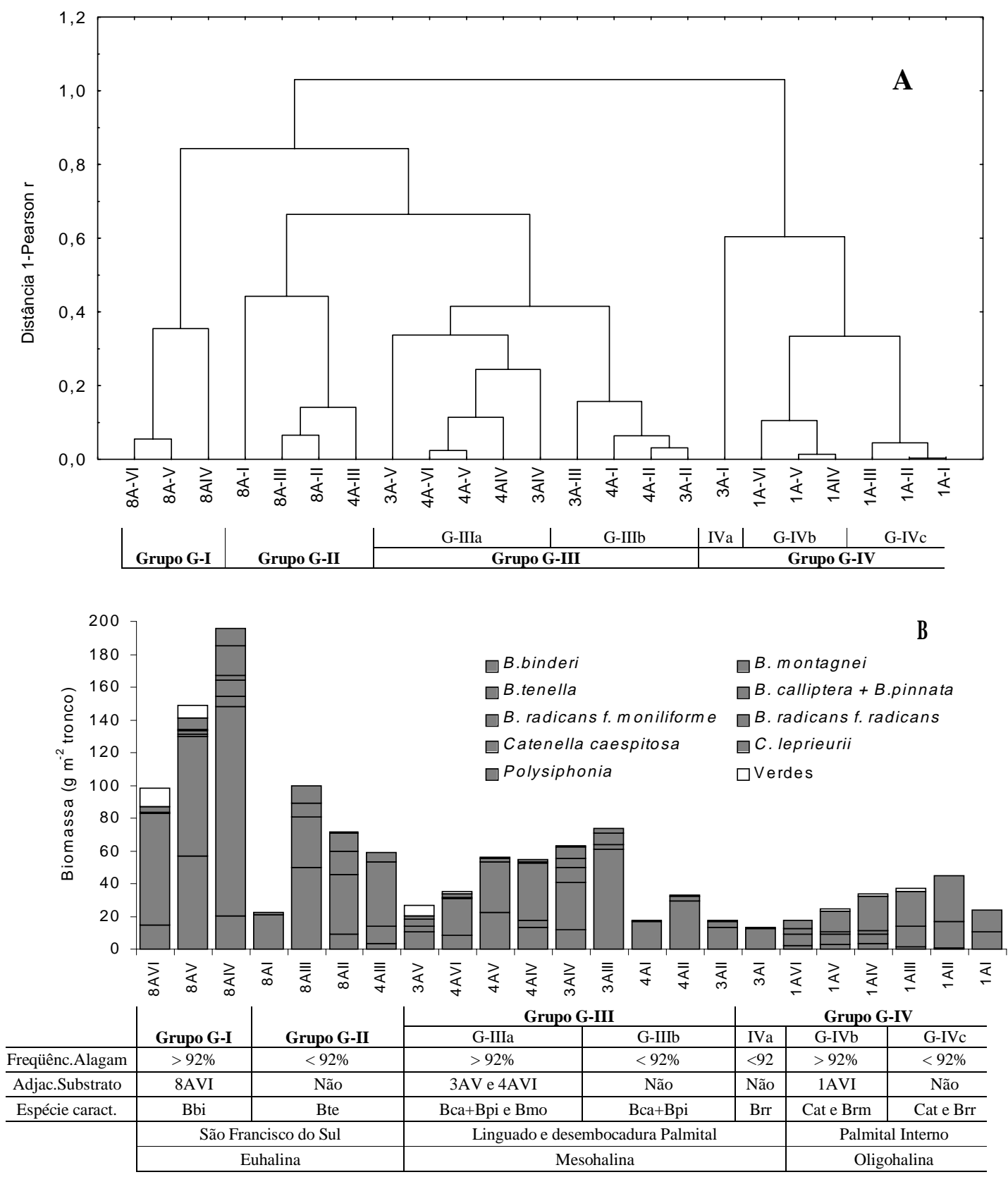

Figura 4: (A) Agrupamento das comunidades de macroalgas associadas a troncos de manguezais (1-r de Pearson) e sua relação com a posição ao longo da Baía da Babitonga (estações 1, 3, 4 e 8) e com a freqüência de alagamento (níveis I a VI) no verão de 1998. (B) Para cada grupo são apresentadas a biomassa por espécie ( $\mathrm{g} \mathrm{ms} \mathrm{m}^{-2}$ tronco) em cada nível de freqüência de alagamento, a espécie característica do grupo (Bca: B.calliptera; Bmo: B.montagnei; Bpi: B.pinnata; Brm: B.radicans f.moniliforme; Brr: B.radicans f.radicans; Bte: B.tenella; Cle: C.leprieurii; Cat: C.caespitosa) e o nível de cada estação localizado adjacente ao substrato. 
do sobre a variação espacial da biomassa e da diversidade das macroalgas de manguezal: a variação da salinidade e da freqüência de alagamento.

A variação da salinidade justificaria a variação da comunidade e da biomassa ao longo da baía. A substituição na dominância da comunidade de macroalgas associadas às porções dos troncos sujeitas a alta freqüência de alagamento, de B.montagnei na região euhalina (mais favorável às espécies de origem marinha), por B.calliptera e B.pinnata na mesohalina e destas por Catenella caespitosa na oligohalina, sugere que esta seqüência reflete uma hierarquia competitiva. Embora estes resultados devam ser considerados com ressalvas, uma vez que estão baseados em uma única amostragem de verão, estes foram consistentes com os dados observados na estação (Cunha, 2001), que sofreu acompanhamento mensal, e com os aspectos teóricos discutidos acima, o que permite assumir como válido o padrão observado ao longo da Baía da Babitonga neste trabalho.

Considerando a variação das comunidades com relação à freqüência de alagamento, o conceito de nível crítico de maré é um conceito antigo (Colman, 1933 e Doty, 1946, in Lobban \& Harrison, 1994) que algumas vezes pode ser útil na explicação dos padrões de zonação das zonas intermareais, embora seja bastante criticado por não considerar a importância dos aspectos bióticos na zonação (Lobban \& Harrison, 1994).

Os níveis críticos de maré são aqueles pontos nos quais acima e abaixo deles há uma marcada modificação na duração da emersão e submersão (Lobban \& Harrison, 1994). Embora haja amplas discussões sobre a validade dos níveis críticos de maré, ao comparar a comunidade de macroalgas associadas a troncos de manguezais ao longo da Baía da Babitonga, foi possível observar na Análise de Agrupamento (Figura 4A e 4B) que em cada uma das estações da baía há uma separação da comunidade em dois grupos, sempre em uma mesma freqüência de alagamento: 92\% (nível alagado durante a maré alta em $92 \%$ dos dias). Acima desta freqüência de alagamento a comunidade está sujeita a exposição contínua por 5 a 8 dias. Abaixo desta, a comunidade está sujeita a exposição contínua somente por 1 a 3 dias.

Embora ao considerar apenas a composição específica não seja possível verificar este padrão com clareza devido ao fato de praticamente todas as espécies estarem distribuídas ao longo de toda a extensão vertical do tronco, quando consideramos a biomassa de cada espécie, o padrão de zonação fica mais evidente. Algumas espécies só apresentaram elevada contribuição para a biomassa total em níveis sujeitos a freqüências de alagamento superiores a $92 \%$. Outras espécies só apresentaram elevada contribuição para a biomassa total em freqüências de alagamento inferiores a $92 \%$. Além disto, em freqüências de alagamento superiores a $92 \%$, um número maior de espécies contribuiu para a biomassa total do nível.

Como este valor de freqüência de alagamento foi um ponto de corte comum a todas as estações, é possível assumir que, para as macroalgas associadas a troncos de manguezais na Baía da Babitonga, o conceito de níveis críticos de maré é funcional, e uma emersão contínua maior ou menor que 4 dias pode ser assumida como crítica para a comunidade.

\section{CONCLUSÕES}

A composição específica das macroalgas associadas aos troncos e pneumatóforos dos manguezais da Baía da Babitonga foi similar à flora algal tipicamente observada em manguezais.

Tanto a biomassa, como a estrutura da comunidade de macroalgas associadas a troncos, sofreram forte influência dos gradientes de salinidade ao longo da baía. 
A comunidade foi mais diversificada e apresentou maior biomassa na porção euhalina da baía.

Há uma freqüência de alagamento crítica para a comunidade de algas de troncos, o que ressalta a necessidade de estudos envolvendo respostas das algas à dessecação, tanto em termos de tolerância como de produtividade e taxas de reposição de biomassa.

\section{REFERÊNCIAS BIBLIOGRÁFICAS}

Aikanathan, S. \& A. Sasekumar. 1994. The community structure of macroalgae in a low shore mangrove forest in Selangor, Malaysia. Hidrobiologia, 285:131-137.

Bertness, M.D. 1999.The ecology of Atlantic Shorelines. Sinauer Associates, Inc. Publishers. 417 pp.

Bouzon, Z. L. \& L.C. Ouriques. 1999. Occurrence and distribution od Bostrychia and Caloglossa (Rhodophyta, Ceramiales) in the Ratones River mangrove, Florianopolis - SC - Brazil. Insula, 28:4352.

Broderick, M.E. \& C.J. Dawes. 1998. Seasonal photosynthetic and respiratory responses of the red alga Bostrychia tenella (Ceramiales, Rhodophyta) from a salt marsh and mangal. Phycologia, 37(2):9299.

Cunha, S.R. 2001. Estrutura e produção das comunidades de Macroprodutores dos manguezais da Baía da Babitonga, SC. Universidade do Rio Grande.

Cutrim, M. V. J. 1998. Distribuição espaço-temporal de macroalgas em troncos e pneumatóforos de Avicennia germinans (L.) Stearn em duas áreas de mangeuas da llha de São Luis - MA, Brasil. Universidade de São Paulo, 158.

Dawes, C. J. 1998. Marine Botany. $2^{\text {nd }}$ Ed. Wiley Interscience publication. 480 pp.

Dawes, C.J. 1996. Macroalga diversity, standing stock and productivity in a northern mangal on the west coast of Florida. Nova Hedwigia, 112:525-535.

Dawes, C.J. 1996. Macroalga diversity, standing stock and productivity in a northern mangal on the west coast of Florida. Nova Hedwigia, 112:525-535.

Doty, M.S. 1946. Critical tide factors that are correlated with the vertical distribution of marine algae and other organisms along Pacific Coast. Ecology, 27:315-328.

Eston, V.R.; Braga, R.M. A.; Cordeiro-Marino, M.; Fujii, M.T. \& N.S. Yokoya. 1992. Macroalgal colonization patterns on artificial substrates inside southeastern Brazilian mangroves. Aquatic Botany, 45:315-325.

IBAMA, 1998. Proteção e controle de ecossistemas costeiros: manguezal da Baía da Babitonga., 146 p.

Karsten, U.; Koch, S.; West, J. A. \& G. O. Kirst. 1994a. The intertidal red alga Bostrychia simpliciuscula Harvey ex J. Agardh from a mangrove swamp in Singapore: acclimation to light and salinity. Aquatic Botany, 48, 313-323.

Karsten, U.; West, J. A.; Zuccarello, G. C. \& G. O. Kirst. 1994b. Physiological ecotypes in the marine alga Bostrychia radicans (Ceramiales, Rhodophyta) from the East Coast of the USA. Journal of Phycology, 30, 174-182.

Lobban, C. S. \& P. J. Harrison. 1994. Seaweed ecology and physiology. Cambridge University Press. 366 pp.

Mann, F.D. \& T.D. Steinke. 1988. Photosynthetic and respiratory responses of the mangrove-associated red algae, Bostrychia radicans e Caloglossa leprieurii. South African Journal of Botany, 54:203207.

Peña, E.J. 1998. Phisiological ecology of mangrove- associated macroalgae in a tropical estuary. Ph.D. Thesis. University of South Carolina. 259p.

Peña, E.J.; Zingmark, R. \& C. Nietch. 1999. Comparative photosynthesis of two species 
of intertidal epiphytic macroalgae on mangrove roots during submersion and emersion.Journal of Phycology, 35:12061214.

Phillips, A.; Lambert, G.; Granger, J. E.; \& T. D. Steinke. 1996. Vertical zonation of epiphytic algae associated with Avicennia marina (Forssk.) Vierh. pneumatophores at Beachwood Mangroves Nature Reserve, Durban, South Africa. Botanica Marina, 39:167-175, 1996.
Post, E. 1968. Zur Verbreitungs-Okologie des Bostrychietum . Hydrobiologia, 31:241316. Raffaelli, D. \& Hawkins, S. 1996. Intertidal ecology. London, Chapman \& Hall. $356 \mathrm{pp}$.

Yokoya, N.S.; Plastino, E.M.; Braga, R.M.A.; Fujii, M.T.; Cordeiro-Marino, M.; Eston, V.R. \& J. Harari. 1999. Temporal and spatial variations in the structure macroalgae associated whith mangrove trees of llha do Cardoso, São Paulo state, Brazil. Revista Brasileira de Botânica, 22(2):195-204. 